The commonest inflammatory condition of the colon is diverticulitis superimposed on a diverticulosis. The diagnosis of diverticulosis is made by the appearance of diverticula formation seen after an opaque meal. In the early stages of diverticulosis there is evidence of breaking up of the haustral segmentation of the colon, and this is followed later by small bead-like projections of the bowel. A superimposed diverticulitis is always associated with a palpable mass at the site of a narrow irregular defect of the bowel. The lesion usually extends further along the axis of the bowel than does a malignant defect. It is extremely difficult to detect a malignant defect which is superimposed on a diverticulosis.

\section{Lesions Outside the Wall.}

The commonest lesion to involve the bowel from outside the wall is an abdominal tumour, and the most common cause is an ovarian cyst. The bowel will be found to be displaced by the tumour mass to which it is bound down by adhesions. Rarely do other tumours involve the bowel, though they frequently cause displacement. Pancreatic tumours will displace the bowel forwards and will compress the duodenum. They are readily recognised, particularly by the duodenal displacement, in the lateral view, and by the splaying out of the duodenal loop. Renal tumours rarely cause displacement and compression.

Obstruction by volvulus is usually recognised in the acute stage. If sub-acute the presence of very mobile bowels and redundant looping of the colon will aid diagnosis of this condition.

Obstruction in a hernial sac is recognised by the presence of the hernial sac containing loops of bowel. The radiograph will show the bariumfilled bowel in an abnormal situation.

Intussusception is rarely sent for diagnosis radiologically as it is easily recognised clinically. In the adult; however, it does sometimes result from the presence of a papillomatous mass. By the enema the rounded end of the apex of the intussusception is recognised, and the mass palpable behind this will confirm the diagnosis. After evacuation of the enema barium will be found in the loop of bowel forming the intussusception.

\section{Lesions of the Nervous Control of the Bowel.}

I.e. Paralytic ileus and achalasia of the pelvirectal sphincter (Hirschprung's disease). These are distinguished by the marked dilatation of the bowel which accompanies these legions.

In paralytic ileus the whole abdomen will be seen filled with widely distended loops of small bowel, and rarely will fluid levels be seen. In Hirsch- prung's disease the appearance of the colon depends on whether the colon is loaded with faeces or distended with gas.

In this condition the colon becomes markedly dilated as far back as the caecum particularly in long established cases. The narrowing of the colon is at the pelvi-rectal junction and is quite regular. The colon retains its haustration till late in the disease, despite the dilatation.

Finally, the correlation of the clinical findings and the radiological appearances must be considered before a final diagnosis is made. The time spent by the radiologist in investigating and localising the site of the lesion before operation will be amply repaid in time saved for the surgeon.

\section{PHYSIOLOGICAL MECHANISMS IN INTESTINAL OBSTRUCTION AND ITS TREATMENT \\ By Charles Reid, M.A., M.D., D.Sc.(Aberdeen), D.P.H.(Cambridge) \\ (Reader in Physiology in the University of London at the London Hospital Medical College)}

\section{INTRODUCTION}

It is probably true to say that physiologicas principles should provide the basis for medical and surgical treatment as far as possible. Tor agree with this statement does not mean that we lose sight of the individuality of the individual, the value of empiric treatment, or the effect that projection of a highly developed nervous system can have on the functions of many of the tissues of the body. Nevertheless, it should be a problem of considerable interest to review the physiological principles involved in the symptomatology and treatment of intestinal obstruction.

\section{INTESTINAL OBSTRUCTION}

Intestinal obstruction may come on suddenly and be complete, or it may come on more slowly 3 and eventually become acute or complete. Furthermore, the obstruction can occur in any part of the intestinal tract, and, as we shall see later, the urgency of the condition decreases as the obstruction is sited near the distal part of the gut.

The causes of intestinal obstruction are many. No doubt obstruction occurs more frequently in certain sites than in others, and the causes thereof may be linked with anatomical factors and, very rarely, developmental factors. If one may generalise, obstruction is due to intrinsic or extrinsic mechanisms:

(I) In the wall of the gut itself, e.g. neoplasm. 
(2) From a foreign body in the gut.

(3) Through an abnormal state of irritability or the musculature causing spasm or atony of intussusception.

(4) Through extrinsic factors such as adhesions, tumours, strangulation of a hernia, tuberculosis affecting the peritoneum.

(5) Through interference with its blood supply, e.g. by embolus.

(6) Through disturbance of the normal nervous control of the bowel, e.g. Hirschsprung's disease.

\section{THE RESULTS OF INTESTINAL OBSTRUCTION} Vomiting.

The immediate results of intestinal obstruction vary with the site of the obstruction and the rapidity with which the obstruction becomes complete. If the stoppage is near the proximal part of the small intestine, vomiting, pain and shock appear early and are severe. On the other hand, if the obstruction occurs low down in the large bowel, the condition is less acute and insistent. The proximal part of the small gut has a higher metabolic rate than the distal part and no doubt the higher rate of production of contraction waves and of secretion of the former, coupled with the closer sensory association of the upper part of the gastro-intestinal tract with the vomiting reflex, is the decisive factor. Thus abnormal tension can develop in the proximal part of the small intestine, and the nervous impulses thus set up can be added to by other impulses produced by the retrograde escape of intestinal fluid into the stomach. If vomiting is severe and prolonged the phenomena associated with dehydration are observed.

The early vomiting in intestinal obstruction removes the gastric contents at first and then the intestinal contents, i.e. an acid fluid and one near the neutral point are lost. Later in continued vomiting, presumably the subject is losing mainly the alkaline secretions from the intestine plus the slightly acid fluid derived from the action of bacteria on proteins and other substances of the intestinal contents. The net result of vomiting in obstruction would appear to be a lowering of the total alkali reserve of the organism-a phenomenon of some importance especially in view of the incidence of acidosis in conditions of shock.

\section{Pain.}

The early pain of intestinal obstruction is due to abnormal activity of the proximal part of the gut and the rising tension therein. Later, intestinal muscle becomes adapted to prevailing conditions, the contraction waves decrease in frequency and force and the muscle becomes quiescent during gradually increasing intervals of time. With the onset of reactive changes in the peritoneum in close relation to the obstructed portion of the gut more pain receptors become involved. The pain changes its character, viz. from periodic attacks of acute colicky pain to a more or less steady dull pain and feeling of discomfort. The abdomen is also tender to pressure.

The localisation of the initial pain should help to determine the site of the obstruction. An obstruction in the duodenum might cause acute pain like that felt in the acute spasms often associated with duodenal ulcer or duodenitis. Again, obstruction near the termination of the ileum or of the colon would induce the sensation of pain commonly associated with other lesions in these situations. However, localisation of abdominal pain in obstruction is secondary to diagnosis and the institution of measures for its relief, and, in any case, the pain which develops later is of little significance for localisation of the site of the obstruction.

\section{Shock.}

The intestinal blood vessels have important vasomotor connections. In addition a large vascular area can be involved in intestinal lesions, and it is hardly surprising therefore to find that shock is always a prominent symptom of intestinal obstruction.

The neurogenic origin of shock is questioned nowadays, but there is no doubt that manipulation of the intestines during abdominal operations disturbs the mechanism controlling the cardiovascular and respiratory systems. Thus it appears likely that the initial stimulation of the gut resulting from the obstruction could induce cardiovascular disturbances by neurogenic mechanisms. Later, no doubt, congestion of the intestine is associated with loss of fluid into the intestinal wall and mesentery, and to a much less degree into the intestinal lumen, a loss of body fluid increased by vomiting. Later, toxaemia develops, and so another factor accentuating the state of shock is introduced. Moreover, it has been claimed that, following strangulation of a short loop of the ileum or obstruction at other sites, the local loss of fluid and the resulting deficit in the total circulating fluid and plasma protein are sufficient not only to account for the ensuing fall in blood volume and in the blood pressure, but also to produce death. These considerations leave aside the possibility not yet demonstrated that the damaged wall of the bowel may release a muscle shock factor, such as has been claimed in traumatisation of skeletal muscles, but they emphasise the importance of anti-shock measures. 


\section{Paralytic Ileus.}

Paralysis of the bowel follows obstructionapart altogether from the temporary paralytic ileus which follows abdominal operations. Violent propulsive waves occur after obstruction on the proximal side of the gut, but in time adaptation occurs. The intestinal muscle accommodates itself to the increasing fluid and gaseous contents of the bowel and the tension in the bowel may rise to or exceed the capillary pressure. With conditions favouring decreased capillary blood flow, necrosis of the bowel wall becomes imminent, although the bowel wall has considerable powers of resisting partial ischaemia. It is likely, moreover, to be damaged by the prevailing toxaemia, and, in consequence, its powers of recovery seriously diminished.

\section{Toxaemia and Peritonitis.}

In the ordinary way the passage of bacteria into the blood or at least the passage of notable numbers of micro-organisms is prevented by the normal integrity of the intestinal mucosa. In addition, wandering cells or phagocytes, which pass to and fro across the mucosa, also play a part as well as the numerous lymphocytes and phagocytes which are freely found in the intestinal mucosa. On the other hand, the mucus-secreting cells which line the gut form a mechanical barrier to microorganisms. In obstruction the resistance or vitality of the intestine deteriorates not only at the site of the obstruction, but also in the area of high intra-intestinal pressure. Under these conditions bacteria invade the damaged tissues and soon inflammatory and infective conditions extend to the peritoneum.

\section{Failure of Nutrition.}

On theoretical grounds failure of nutrition of the organism could occur through persistent vomiting, toxaemia, loss of digestive and absorbing surfaces, toxic anorexia and toxic hepatitis. In the pre-operative period failure of nutrition is not likely to be prominent. On the other hand, the maintenance of the nutrition and incidentally the resistance of the individual should be a matter of concern during persisting post-operative ileus or toxaemia.

\section{The Functioning of the Liver.}

Liver function is essential to maintain the life of the individual. It is true that the functional reserve of the liver is large. Nevertheless, under the stress of an abdominal emergency, in which lowered intake of essential foodstuffs is bound to occur, and in which the liver must receive directly via the portal system the blood draining the damaged and infected intestine, it would be $\stackrel{0}{0}$ interesting to know the behaviour of the efficiency of the liver as judged by the newer tests of hepatic $z$ function.

\section{TREATMENT}

Treatment in intestinal obstruction may be con- $\frac{\vec{F}}{\underline{0}}$ sidered from the following standpoints: (I) urgency $\frac{\overrightarrow{0}}{0}$ of the condition, (2) pre-operative, (3) operative, (4) post-operative.

\section{Urgency}

All cases of obstruction in which the viability of a portion of the intestine is involved should be dealt with at the earliest possible moment by nonoperative measures for the relief of the obstruction or by operation. It is rare that a strangulated $\stackrel{\omega}{\omega}$ hernia is relieved by non-operative measures such $\overline{8}$ as taxis, but these may be attempted while the 3 . patient is being prepared for operation. Where the obstruction is thought to be due to a tumour $\vec{\sim}$ affecting for example the distal part of the large $\underset{N}{\sim}$ bowel, it is usually justifiable, if the obstruction 0 is recent and the patient's condition allows, to wait for a confirmatory examination. Meanwhile the oedema of the bowel which aggravates a partial obstruction and depresses the motility of the gut may disappear under suitable treatment for the relief of the distension. The general condition of the patient and the local state of the bowel may both improve and so permit more satisfactory conditions for operation.

\section{Pre-operative Treatment}

Apart from the considerations raised in the preceding paragraph and apart from routine general measures, it may be a matter for discussion as to whether measures to deal with shock or to prevent or mitigate the subsequent development of shock should be instituted at this stage. With this end in view it appears justifiable to give morphine which quietens the patient and inhibits the propulsive waves of the gut. The quiescence of the gut might prevent or decrease the loss of fluid into the bowel wall and so decrease the likelihood of further depletion of the blood volume. More direct anti-shock measures might be adopted with advantage in cases when immediate operation is to be attempted. It appears sound, therefore, to give plasma and saline before operation, but there may be practical objections to this which are not immediately evident.

If the radical operation for the relief of obstruction can be delayed, suitable diet, including a high proportion of its protein as first-class protein, must be given because the resistance of the patient to shock and probably infection depends on the building up of a generous protein reserve in the liver. 


\section{Operative}

The field of operative technique for the relief of intestinal obstruction belongs properly to the surgeon. The immediate and post-operative success alike depends on the excellence of his technique and judgment as to what should be done to deal with conditions met after the abdomen is opened. In strangulation of the bowel resection of a part of the bowel may be required. Then the surgeon is guided as to the viability of the gut by the interval of time since strangulation and the appearance of the wall of the bowel. One or two tests of viability may be tried. The application of warm saline packs for five minutes restores tone if the gut is viable, while the administration of pure oxygen to the patient by raising the oxygen tension in the tissues causes a viable portion of gut to regain its normal pink colour. Further, the length of gut resected is not of vital importance, as a few feet of small intestine can provide adequate digestive and absorptive surfaces to maintain nutrition when the diet is chosen with judgment. Accordingly it is not justifiable to retain a piece of gut whose viability is not confirmed.

On theoretical grounds an end-to-end anastomosis would be expected to give as good afterresults as a lateral one. In neither case can a contraction wave pass across the area of union so that propulsion in the distal segment must depend on the initiation of a new contraction wave by the response of its musculature to stretch.

In practice the choice of the method of union appears to depend on the individuality of the surgeon. An end-to-end anastomosis may present difficulties owing to inequality in calibre of the proximal and distal ends, but these can be overcome.

The successful solution of problems of surgical technique in intestinal obstruction, even if eminently successful, must be of no avail unless they are accompanied by measures designed to deal with present or expected complications. The risk of infection is lessened by careful stitching of the bowel, which prevents soiling of the peritoneum, and by removal of excess of fluid from the abdominal cavity by suction. The latter procedure avoids disturbance of the self-protective mechanisms of the peritoneal tissues. To arrange for peritoneal post-operative drainage may likewise be necessary. Probably, however, the most urgent condition requiring remedial measures is distension, which if severe may obliterate blood flow in the capillaries. Obviously unless the intra-intestinal pressure is lessened or kept below capillary pressure the intestinal muscle will not recover its irritability. Therefore anti-distension measures are of supreme importance, and, when necessary, they should be instituted during the operation to relieve the pre- vailing distension if severe, and to prevent the onset of further distension in the post-operative period. The "most physiological" method is enterostomy with the insertion of a small rubber tube. By this tube continuous drainage of the intestine can go on, distension is relieved, and, when required, suitable volumes of nutrient fluids can be run in and retained for short periods to allow of digestion if necessary and absorption of the foodstuffs. Duodenal and rectal tubes can be used but, if the obstruction affects the small intestine, it is clear that neither duodenal nor rectal intubation is certain to relieve the distended loops. Apart from this point, which is an important one, there are other objections and limitations to their use.

When the intestinal obstruction is due to a tumour the lesion is usually found in the large intestine. Oedema of the bowel wall may aggravate a partial obstruction and cause an unnecessary degree of distension. In this case it is always justifiable to delay the radical treatment. The first stage aims at relieving the distension by colostomy. Thereby the bowel wall is allowed to attain a more suitable state for the subsequent radical operation. Apart from the local improvement, however, the general condition of the patient improves after relief of the obstruction and reduction of the toxaemia.

The clinical condition of megacolon (Hirschsprung's disease) resembles that of a chronic obstruction of the colon and apparently shows a lack of balance between the sympathetic, which is dominant, and the parasympathetic nerve supply to the bowel. If the lack of balance is the complete explanation of the condition, treatment to be successful should aim at sympathetic denervation of the bowel and perhaps of the adrenal medulla.

\section{Shock.}

\section{Post-Operative}

A moderate state of shock is always a feature of the immediate post-operative period after surgical treatment of intestinal obstruction. The operation itself adds to the conditions which have favoured its development from the onset of the obstruction. The question as to the propriety of adopting appropriate anti-shock measures then arises. As is well known, shock is characterised by the relative inadequacy of the cardio-vascular mechanism and by a diminished blood volume. There is in addition a low metabolic rate with an accompanying fall in the body temperature. Moreover, a tendency to acidosis develops as a result of diminished kidney function and of anaerobic glycolysis in the anoxic tissues releasing lactic acid and so diminishing the alkali reserve. The 
inadequacy of the circulatory mechanism may be correlated with the loss of fluid chiefly into the bowel wall and mesentery. It is of interest to note that the lost fluid in experimental trauma of the intestine contains at first large amounts of albumin and the total loss of albumin may be larger than is present in the circulating plasma. The source of albumin must be mainly the liver by virtue of the dynamic equilibrium which exists between the tissue (liver) albumin $\rightleftharpoons$ plasma albumin. This point emphasises the importance of a high standard of nutrition in individuals who become operative risks.

Anti-shock measures need not include the transfusion of whole blood unless the usual indications for it are present. A physiological "RingerLocke" solution containing normal amounts of plasma protein might be the most suitable fluid for infusion. There are, however, objections to this form of infusion, and it may be that the best response of the shocked subject is obtained by the intravenous infusion of concentrated serum or despeciated bovine serum and the exhibition of normal saline into a vein or by other channels. On theoretical grounds it appears sounder to use a "Ringer-Locke" solution containing appropriate concentrations of glucose and bicarbonate.

Another form of therapy which might be worth investigation is the exhibition of vitamin $\mathrm{BI}$ to the shocked patient. In conditions of shock in animals (dogs) due to haemorrhage and of anoxia, much of the cocarboxylase of the tissues becomes dephosphorylated:-

Vitamin BI + Phosphate

$$
\frac{\text { aerobic }}{\frac{\text { pyrophosphatase cocarboxylase }}{\text { anaerobic }}}
$$

i.e. anaerobic conditions shift the position of equilibrium to the left. The administration of vitamin $\mathrm{BI}$ to shocked animals is apparently beneficial. Provided the tissue pyrophosphatase retains activity, the above seems a rational form of therapy in view of the many intracellular reactions which depend on cocarboxylase.

\section{Paralytic Ileus.}

Inhibition of intestinal motility is probably the most serious sequela of intestinal obstruction and its treatment. Simple section and anastomosis of the bowel, it should be remembered, inhibit intestinal motility for at least two or three days. Moreover, the distension due to accumulation of fluid and gas may produce ultimately a tension sufficiently high to prevent contraction of the intestinal muscle and obliterate the capillary blood flow. Further, the concomitant toxaemia intro- duces another factor which depresses the reactivityo of the intestinal muscle. Measures should aim at relieving distension by drainage and diminishing 3 toxaemia. The one sure method for the relief of $\frac{\widehat{\Phi}}{2}$ distension and of the ileus is drainage of the $c$ proximal distended segments so that eventually $\vec{\Rightarrow}$ the muscle recovers its irritability and reacts to $\stackrel{\mathcal{P}^{2}}{ }$ the normal stimuli. In cases where intestinalo motility is not completely lost or is returning, it may be justifiable to attempt to promote motility $\frac{w^{n}}{2}$ by the use of drugs. Post-pituitary extract $\stackrel{\mathbb{\perp}}{\circ}$ prostigmine and ergotamine tartrate in combination are said to be most effective in favouring the propagation of propulsive waves. On the other. hand, a drug may be preferred such as carbaminoyl $\vec{\omega}$ choline chloride (Doryl or Moryl), which is more $\stackrel{\circ}{\circ}$ closely allied to the normal impulse transmitter of: the parasympathetic nerve endings in Auerbach's 3 . plexus. During the period of non-reactivity of the bowel wall when distension is severe or infection $\vec{i}$ of the peritoneum is at its height, treatment by drugs is useless as they may produce vicarious 0 spasm without co-ordinated peristalsis. Drugs $\stackrel{\circ}{\circ}$ can be used with justifiable results only when $?$ irritability and contractility have returned to the $\vec{z}$ recovering muscle, for they cannot initiate contraction waves in intestinal musculature which has for the time being lost its irritability.

\section{Infection and Toxaemia.}

Measures adopted at operation for the relief $\overline{\text { off }}$. distension, careful suture and peritoneal drainage (if soiling has occurred), should limit and ulti-o mately diminish infection. The patient's resist-命 ance is all-important as specific treatment byํ․ vaccines or anti-sera has proved disappointing. $\overrightarrow{\overrightarrow{0}}$ 'In recent times, however, other therapeutic agents 3 have become available and opportunities may? occur for evaluating their use following intestinalo obstruction and its treatment.

The administration of fluids is usually one of 3 the measures adopted to deal with toxaemia. It is $\sigma$ claimed that "the administration of large volumes of normal saline into a vein or under the skin haso given good results; 4 to 6 litres should be given in₹ the first 24 hours and not less than 2 to 3 litres ono the third day. No fluid is given by mouth and this, combined with drainage from the enterostomyo tube, - ensures against overloading the patient with fluid." The rationale of this form of hydro-therapy is not immediately apparent. No doubt ${ }^{N}$ treatment on these lines combined with the N injection of plasma proteins must aid the reactive ${ }_{\sigma}$ powers of the organism by helping to maintain the circulation, and, in any case, the injected protein, if not required in the circulatory fluids of $\stackrel{9}{\rightarrow}$ the body, can 'be utilised by the organism provided 0 the liver's efficiency has not deteriorated. Theo 
basis for the latter part of the preceding statement depends on the experiment in which a dog was kept in a state of nitrogenous equilibrium by giving homologous plasma into a vein and carbohydrate and fat by mouth. It is unlikely that dilution per se of the body fluids by a few litres of saline is important in dealing with the toxaemia. The saline infusion however will induce diuresis, and, provided the toxins consist of molecules sufficiently small, e.g. molecular weight about 60,000 or less, to pass through Bowman's capsule, relief of the toxaemia might thus be effected. It should be noted that the excretion of urea, a nontoxic normal metabolite, increases or decreases generally with the volume of the urine which is formed; it is "filtered" from the blood in the glomeruli and partially reabsorbed in the tubules to a variable extent, depending on the need of the organism to conserve water. Further, tubules of the kidneys might actively excrete the toxins in the same way.as they remove foreign substances, such as diodrast or phenol red, from the blood. If this is the case the need for a decided diuresis is not so obvious. In the absence of information concerning the removal of toxins in the urine a decision as to the manner in which saline infusions are said to mitigate toxaemia must be withheld.

Another possibility is perhaps worthy of consideration. In toxic conditions the adrenal cortex may be affected so that the output of its essential hormone is lowered and the normal electrolytic balance in the tissues is disturbed. Furthermore, the plasma potassium may be increased not only by the escape of potassium from tissues damaged by the toxaemia and the operation, but also by the peripheral action of adrenaline, secreted by the adrenal medulla in the emergency. Now it is known that the exhibition of a normal salt mixture prolongs the life of adrenalectomised animals more or less indefinitely even in the absence of the administration of the cortical hormone. It is possible that the beneficial effect of saline infusions or better still of balanced salt mixture depends on the restoration of the normal electrolytic balance in the tissues. The adrenal cortical hormone is of course not a suitable agent for the treatment of shock in general.

\section{Feeding.}

The problem of feeding is complicated by the almost inevitable presence of intestinal stasis and anorexia. In view of the fact that intestinal motility increases the blood supply to the mucosa and its secretory activity, the digestive mechanisms are certain to be depressed in stasis. There is thus little use in feeding food which is not readily digested. In fact the conditions seem prerequisite for the provision of predigested foods. If this principle is adopted, the necessary first-class proteins including meat can be hydrolysed to their constituent amino-acids, carbohydrate given in the form of glucose, and fat hydrolysed by lipase in the presence of bile salts. In this way a soluble and absorbable mixture with the addition of vitamins can be given by the enterostomy or duodenal tube. Rectal feeding is entirely useless as the large intestine is not designed either for digestion or for active absorption. The epithelium of the colonic mucosa is simple and relatively undifferentiated as compared with that of the small intestine. Moreover, rectal or colonic feeds of unhydrolysed foods are not absorbed and, even when predigested foods are given by this route, absorption or rather diffusion of glucose or aminoacids across the mucosa is slow.

Ideally, no doubt, the best method of feeding is to infuse protein or a protein hydrolysate into a vein along with glucose. The gut is thus left completely at rest and further secretion is greatly reduced, so that formation of unabsorbed residues from the intestinal mucosa is reduced to a minimum and the distension caused by the action of bacteria on the intestinal contents is greatly reduced. There may be technical and practical objections to the above procedure, but its possibilities are worth exploring for use in severe cases of paralytic ileus.

Finally, if absorption from the gut is in doubt, it is still possible to give protein into a vein either as plasma or despeciated bovine serum. On the whole, enzymatic hydrolysates of protein are not well tolerated when given into a vein, but they can be replaced by a mixture of the ten essential amino-acids of Rose in appropriate concentrations, to which may be added glycine in order to improve the subject's tolerance. The chief drawback to this form of feeding is the expense, but the treatment would not be required for more than a few days.

\section{Restoration of the Normal Bowel Action.}

Eventually in a recovering case of paralytic ileus a massive action of the bowel is obtained, aided perhaps by the timely exhibition of parasympathetic drugs such as carbaminoyl choline chloride. Constipation, however, is liable to be rather troublesome, especially as the subject is confined to bed, is fed on a bland diet, and the usual signal to reflex defaecation is temporarily in abeyance. One might put forward here and now a plea for the physiological treatment of the bowel mucosa and the avoidance of irritant pharmacological activation by adding to the diet of the convalescent a non-absorbable material which retains moisture and so adds bulk to the faeces. This method, coupled with an attempt to link the 
normal reflex mechanism again with the act of defaecation and a minimum usage of the inhibitory bed-pan, should lead quickly to the re-establishment of normal bowel action.

It gives me pleasure to state that I have valued greatly the suggestions made by Mr. H. Freeman, F.R.C.S., who kindly vetted my manuscript, particularly where it impinged on clinical medicine and on surgical technique.

\section{SCLEROTIC THERAPY IN PRACTICE III}

\section{HAEMORRHOIDS . . . THEIR TREATMENT BY INJECTION}

$$
\text { By R. R. Foote, M.R.C.S. }
$$

The sufferers from piles form a vast legion who frequently receive but little relief from the care of the medical profession. A box of ointment from the general practitioner, or the somewhat doubtful honour of being the last on the operating list of the surgeon (often practice for the new house surgeon) is their not uncommon fate. Twenty-five consecutive patients at my out-patients had not even had the privilege of examination prior to being sent up to hospital . . . why is it that piles are thought to be so boring by the average practitioner?

I hope to show that the cure of haemorrhoids is a very interesting subject, and that the relieved sufferer is not the least grateful of patients. He has, as a rule, hidden his troubles from his doctor for a long time ... he has often become introspective, nervous, and ill from worrying. Rational treatment in the comparatively early stages may save him from continued worry with inevitable surgery later on in life.

Nearly all cases of piles can be relieved by proper injection therapy, and a vast number can be permanently cured. As already mentioned, over 98 per cent of first degree piles remained permanently cured after a five-year period, subsequent to injection treatment at St. Mark's Hospital. A proctologist of great experience told me that he "injected over 70 per cent of pile cases for one reason or another." It is possible that this figure is higher than it should be owing to various factors such as bed shortage, but even taking this into consideration the figures impress the importance of this therapy, which in my experience is so much neglected by the average practitioner.

The contra-indications for this treatment are few and obvious.

I. Inflammation.-Any inflammatory condition associated with piles is an absolute contra-indication to injection. Thrombosis and strangulation must be allowed to settle down before treatment.
2. External piles.-Surgical treatment under local anaesthesia is necessary. The diathermy knife may be used for such cases.

3. Pregnancy.

5. Advanced general disease.

6. The patulous anus with sphincter muscle.

\section{Rectal neoplasm.}

8. Anal stricture.

Relative contra-indications are the presence of advanced third degree piles, and those cases in which a rapid recurrence has followed injection ${ }^{\infty}$ treatment.

The presence of an anal fissure delays the commencement of injection therapy, and in my opinion the presence of an anal fistula negativeso injection. Kasper Blond does not agree with this latter statement, and claims success in a large series of cases in which he has treated the fistulaewith injections followed by sclerosing treatment ofw the piles. I should personally be afraid of the associated sepsis and the risks of septicaemia.

It should be noted especially that the injection treatment is of great value when dealing with badsurgical risks, such as in the cases of the diabetic? consumptive, or senile patient.

The treatment of the sufferer from piles must entail the following considerations:-

I. The taking of a complete and thoroug history.

2. Careful examination, both local and generaț.

3. A proper selection of the case for injection treatment.

4. The right solution to be put into the right place in the right amount.

The only way to learn the details of injection treatment is to do a large number of cases and te learn by means of "trial and error," since the injection of an occasional case will not teach the operator the correct dosage or the finer points technique. Complications in the shape of abscess. formation and septicaemia are not as uncommoig as is generally supposed. The scope of this article is limited by space, and can but serve t@ offer a few suggestions to those who are fortunate enough to be able to gain practical experience at clinic devoted to the subject.

All cases presenting themselves for treatmen\$ should have a very careful cross-examination regarding their symptoms. The questionnairs used at St. Mark's Hospital is very thorough ang will often give a diagnosis before the examination of the patient has been undertaken. The charact\& of the pain, the degree of prolapse, the state of the bowels, and the type and time of haemorrhage together with any pruritic symptoms are af carefully analysed. A general examination is the 